doi:10.1017/S0022215116007064

\section{ID: IP210}

Cartilage versus the rest in Type I

Tympanoplasty - who wins?

Presenting Author: Hsern Ern Tan

Hsern Ern Tan ${ }^{1}$, Peter Santa Maria ${ }^{2}$

${ }^{1}$ 1. Sir Charles Gairdner Hospital, 2. Ear

Science Institute of Australia, ${ }^{2}$ Sir Charles

Gairdner Hospital

Learning Objectives: To determine the efficacy of different graft materials in Type I tympanoplasty through systematic review.

Introduction: In Type I Tympanoplasty, the most commonly used graft materials are temporalis fascia, cartilage and fat, which are all readily accessible at the surgical site. Over the years many other natural and synthetic materials have been trialled, but there are few published studies on outcomes. There has been a renewed interest in cartilage grafting due to its rigidity and resistance to retraction.

Aims: To determine the efficacy of different graft materials in Type I tympanoplasty through systematic review.

Methods: Using a search of the MEDLINE and PubMed databases from 1970 to 2014, all RCTs and retrospective studies reporting the outcomes of Type I tympanoplasty in primary chronic tympanic membrane perforations were identified. The studies were then analysed in a single variable analysis to compare the success rate of tympanic perforation closure between four major graft materials (cartilage, fascia, fat and other).

Results: 214 studies were identified from a total of 4704 abstracts. Cartilage had the greatest success rate of the four groups with $90.80 \%$, across 33 studies (1746 patients) compared to fascia with a success rate of $88.00 \%$, across 121 studies (14806 patients) and significant $p$ value of 0.048 . Fat had a success rate of $86.52 \%$ (across 22 studies and 1507 patients) and the last category of 'other' had a success rate of $85.39 \%$ (across 36 studies, 4217 patients) but the difference was not significant.

Conclusions: Cartilage has a superior graft closure rate compared to fascia in Type I tympanoplasty. Though this is consistent with the findings in recent literature, cartilage is also often used as a graft material for smaller sized perforations, which innately have higher healing rates, and this may account for the increased closure rate with cartilage compared to other graft materials.

doi:10.1017/S0022215116007076

\section{ID: IP211}

Does perforation size matter in myringoplasty?

Presenting Author: Hsern Ern Tan

\author{
Hsern Ern $\operatorname{Tan}^{1}$, Peter Santa Maria ${ }^{2}$ \\ 1. Sir Charles Gairdner Hospital, 2. Ear \\ Science Institute of Australia, ${ }^{2}$ Sir Charles \\ Gairdner Hospital
}

Learning Objectives: The impact of perforation size on tympanic membrane closure in myringoplasty has been well reported in literature. We attempt to summarise the literature findings in a single variable analysis.

Introduction: Graft failure in larger perforations are thought to occur due to increased technical difficult, reduced visibility, reduced graft overlap with the residual tympanic membrane (TM) and a poor vascular bed for graft uptake. However, smaller perforation sizes fail for similar reasons and literature is not conclusive on the significance of perforation size in myringoplasty success.

Aims: The impact of perforation size on tympanic membrane closure in myringoplasty has been well reported in literature. We attempt to summarise the literature findings in a single variable analysis.

Methods: A literature search of all myringoplasty studies from 1966 to 2014 was conducted using PubMed. Retrospective and prospective papers reporting the impact of perforation size and perforation location on graft closure were extracted. A single variable analysis was then performed.

Results: Perforation size greater than $50 \%$ surface area of TM had significantly worse graft closure compared to perforation size less than $50 \%$. Data for perforation size $>50 \%$ came from 58 studies (3374 patients) showing a graft closure rate of $79.44 \%$, compared data for perforation size $\leq 50 \%$ perforation size from 74 studies (5859 patients) showing a closure rate of $85.56 \%$ and a $p$ value of 0.019 . Perforation location (central, anterior or posterior) was not significant though anterior perforations had the least success.

Conclusions: This single variable analysis indicates that in Type I tympanoplasty, perforations greater than $50 \%$ have a lower success rate, while the location of the perforation had no significant effect on success rate.

doi:10.1017/S0022215116007088

\section{ID: IP212}

Combined Endoscopic and Microscopic

Approach to cholesteatoma Surgery

Presenting Author: Jabin Thaj

Jabin Thaj ${ }^{1}$, Praneta Kulloo ${ }^{2}$, Chitta Chowdhury ${ }^{2}$, Gaurav Kumar ${ }^{2}$

${ }^{1}$ Queens hospital, ${ }^{2} T B C$

Learning Objectives: ï Multidiscplinary pre-operative planning. $̈$ ï Modern day Otologist should be proficient in both microscopic and otoendoscopic technique. 